\title{
Les recommandations de la CIPR : les raisons d'un changement
}

\author{
A. SUGIER ${ }^{1}$, J.-C. NÉNOT ${ }^{1}$, J.-F. LECOMTE ${ }^{1}$
}

(Manuscrit reçu le 17 janvier 2005, accepté le 11 avril 2005)

RÉSUMÉ Depuis sa création en 1928, la Commission internationale de protection radiologique (CIPR) produit régulièrement des recommandations sur la protection contre les rayonnements ionisants ; ces recommandations sont habituellement reprises par les organisations internationales et par les États. Depuis 1990, date des recommandations les plus récentes (Publication 60), la progression des connaissances scientifiques, les développements techniques, le retour d'expérience et le souci d'adhérer à l'évolution de la société, ont incité la CIPR à modifier son système de protection. Le tout dernier projet de recommandations, récemment rendu public pour consultation et appel de propositions, est décrit et discuté.

ABSTRACT The recommendations of the ICRP: the reasons for a change.

Since its foundation in 1928, the International Commission on Radiological Protection (ICRP) has regularly produced recommendations on the protection against ionising radiation; these recommendations are currently taken up by international organisations and by states. Since 1990, date of issue of the most recent recommendations (Publication 60), advances in scientific knowledge, technical developments, feedback and desire to meet modern societal developments, have incited the ICRP to modify its system of protection. The latest draft, which was recently presented openly for consultation and proposals, is described and discussed.

Key words: ICRP / recommendations / radiological protection / constraints

Le présent article a pour objectif de situer le projet de nouvelles recommandations de la Commission internationale de protection radiologique (CIPR) dans le cadre de l'évolution de ses travaux et de mettre en évidence, à un moment important du processus de consultation qu'elle a engagé, les principales caractéristiques de ce texte. S'agissant d'un processus en cours, il faut s'attendre à des modifications du texte dans les mois à venir, notamment dans la présentation du tableau des contraintes de dose.

\section{Les bases historiques de la doctrine}

La CIPR a été créée en 1928, quand les radiologues prennent conscience des lésions causées par les rayons $\mathrm{X}$ et par le radium à leurs patients et à euX-mêmes.

\footnotetext{
1 IRSN, B.P. 17, 92292 Fontenay-aux-Roses, France.
} 
La première limite de dose, qui date de 1938, concernait les seuls professionnels et équivalait à environ 500 millisieverts $(\mathrm{mSv})$ par an. Ce système de protection était censé garantir l'absence totale de risque, puisqu'il protégeait contre les effets des fortes de doses de rayonnements, les seuls connus à cette époque. Après la seconde guerre mondiale, l'action cancérogène des rayonnements a été reconnue et il est devenu évident que des expositions inférieures aux limites pouvaient causer des effets extrêmement graves. La CIPR recommande alors un abaissement des limites de dose : $3 \mathrm{mSv}$ par semaine pour les travailleurs (soit environ $150 \mathrm{mSv}$ par an) et le dixième de cette valeur pour la population en raison de possibles risques génétiques et de la sensibilité de certains individus qui les rend particulièrement vulnérables aux rayonnements. La première publication officielle, identifiée comme la Publication 1, date de 1959 (ICRP, 1959) ; la limite professionnelle hebdomadaire laisse la place à une limite annuelle qui tient compte de l'accumulation des doses ; cette limite correspond à une moyenne de $50 \mathrm{mSv}$ par an mais autorise des dépassements exceptionnels, bornés à $30 \mathrm{mSv}$ par trimestre, soit un maximum de $120 \mathrm{mSv}$ par an.

Les publications suivantes sont techniques et explicatives. Dans la catégorie technique, la Publication 2 de 1960 (ICRP, 1960) constitue un document de base pour ce qui concerne l'exposition interne, alors que, dans la catégorie explicative, la Publication $9^{2}$ (1963) jette les bases de la doctrine actuelle, en discutant de l'acceptabilité du risque, puisque que les expositions « habituelles » ne sont pas exemptes de tout risque. À cette époque, la CIPR s'intéresse de très près aux cancers radio-induits et aux effets héréditaires, risques potentiels des faibles doses donc des situations normales ; en revanche, elle délaisse les effets des fortes doses, qui ne peuvent résulter que de situations accidentelles. Le principe d'optimisation de la protection apparaît clairement pour la première fois : maintenir toutes les doses aux valeurs les plus faibles auxquelles l'on peut parvenir sans difficulté, compte tenu des aspects sociaux et économiques (couramment représenté par l'acronyme anglais ALARA : as low as readily achievable) et la limite annuelle est fixée à $50 \mathrm{mSv}$ pour les travailleurs et à $5 \mathrm{mSv}$ pour les personnes du public. L'optimisation de la protection occupe une place de plus en plus importante ; un chapitre entier de la Publication $22^{3}$ (1973) lui est consacré.

En 1977 la Publication 26 (ICRP, 1977) effectue la synthèse des recommandations précédentes, tout en tenant compte de l'actualisation des

\footnotetext{
2 Recommandations de la Commission internationale de protection radiologique, Publication 9 de la CIPR (traduction), 1963. Paragraphe 52. Ed. : Service central de documentation, CEN-Saclay.

3 Recommandations de la Commission internationale de protection radiologique, Publication 22 de la CIPR (traduction), 1973, les implications des recommandations de la Commission de maintenir les doses aux valeurs les plus faibles qu'il soit possible d'atteindre sans difficulté. Chapitre B : Application pratique du paragraphe 52 de la Publication 9, paragraphes 12 à 19 .
} 
connaissances. Elle définit les trois principes de base qui régiront la protection radiologique pendant plus de vingt ans :

- la justification des pratiques,

- l'optimisation de la protection, en reprenant les termes «ALARA »,

- la limitation des doses.

La doctrine de la Publication 26 repose sur une éthique de protection de la collectivité, de type utilitariste ; cette approche considère que, si la protection de la société est assurée, celle de l'individu est aussi assurée de façon satisfaisante ${ }^{4}$. Le système repose sur l' analyse coût-bénéfice, avec comme outils la dose collective, qui est considérée comme une mesure adéquate du risque global en rapport avec une source de rayonnements. La limite annuelle de dose qui garantit la protection individuelle figure au deuxième plan et ne constitue qu'un garde-fou. Elle est maintenue à $50 \mathrm{mSv}$ pour les travailleurs et à $5 \mathrm{mSv}$ pour la population, dans la mesure où la moyenne sur la vie ne dépasse pas $1 \mathrm{mSv}$ par an. La comparaison des risques liés à ces doses avec ceux de la vie courante permet de juger de leur acceptabilité : le niveau de risque est comparable, pour les travailleurs à celui des professions les plus sûres, et pour les personnes du public à celui de la vie de tous les jours. Cette publication évoque en outre la protection de l'environnement : il est probable que le niveau de sécurité nécessaire pour assurer la protection de tous les individus du genre humain convient également pour protéger les autres espèces, sinon nécessairement tous les individus de ces espèces ${ }^{5}$.

\section{La synthèse de la doctrine en 1990}

Dans les années 80 les connaissances sur le risque d'effets stochastiques s'affinent; le risque de cancer radio-induit s'avère sous-évalué et justifie un abaissement des limites. La CIPR entreprend alors un travail de synthèse et de refonte afin d'obtenir un système logique et cohérent, donc d'usage pratique. Ce système est conçu pour garantir une protection de haut niveau à tous les individus dans toutes les circonstances et éviter tout manquement à l'équité. C'est le premier pas vers un recentrage sur l'individu. La Publication 60 (ICRP, 1991) précise d'emblée son objectif ${ }^{4}:$ « procurer à l'homme un niveau de protection approprié, sans limiter indûment les activités bénéfiques à l'origine des expositions ».

\footnotetext{
4 La philosophie utilitariste a été fondée au $17^{\mathrm{e}}$ siècle par Jeremy Bentham, puis répandue au siècle dernier dans les pays anglo-saxons. Contrairement à ce que le mot semble suggérer en français, cette philosophie ne glorifie pas l'égocentrisme, mais constitue une doctrine altruiste. Son principe général peut s'énoncer: une action est bonne quand elle tend à réaliser le plus grand bien possible au plus grand nombre de personnes concernées; ; dans le cas contraire, elle est mauvaise. De façon
plón générale, les utilitaristes considèrent les conséquences économiques bénéfiques qui se rapportent aux différents risques. Ils s'opposent aux tenants de l'éthique contractuelle, qui placent au premier rang le droit à la protection individuelle contre les risques réels ou potentiels.

5 Recommandations 1990 de la Commission internationale de protection radiologique, Publication 60 de la CIPR (traduction), 1991, Pergamon Press, paragraphe 15.
} 
Les trois principes de base sont conservés, mais l'accent est mis sur l'optimisation de la protection, qui constitue le fer de lance du système. L'optimisation s'appuie sur la contrainte, qui se différencie de la limite, car elle s'applique à une source donnée de rayonnements mais est liée à l'individu exposé. La contrainte représente donc une fraction de la limite et constitue une aide à l'exploitant qui doit l'utiliser de façon prospective ; en revanche ce n'est ni une limite supplémentaire ni un niveau d'intervention ou d'investigation. Le système repose toujours sur l'acceptabilité d'un certain niveau de risque, puisque la CIPR considère qu'une relation dose-effet linéaire sans seuil constitue la représentation la plus crédible de l'induction des cancers radio-induits. Pour juger de cette acceptabilité, la CIPR abandonne la comparaison avec d'autres risques, qui le plus souvent n'étaient pas comparables entre eux, en raison de leur nature (par exemple, il est difficile de donner le même poids à un décès aléatoire par cancer survenu quelques dizaines d'années après l'exposition et à une mort accidentelle) et juge de l'acceptabilité du risque radio-induit de façon absolue. Ainsi, en se basant sur un risque de cancer mortel de $4 \%$ par sievert pour les travailleurs (toute personne âgée de 18 à 65 ans) et de $5 \%$ par sievert pour la population (tous âges confondus), la CIPR juge que le maximum tolérable sur la vie entière est 1 sievert pour les travailleurs et $70 \mathrm{mSv}$ pour les personnes du public. Les limites de dose annuelles en sont déduites : abaissées à $20 \mathrm{mSv}$ pour les premiers (avec dépassement autorisé jusqu'à $50 \mathrm{mSv}$ une année, dans la mesure ou la moyenne sur 5 ans ne dépasse pas $20 \mathrm{mSv}$ par an) et maintenues à $1 \mathrm{mSv}^{6}$ pour les seconds. Les limites continuent de jouer le rôle d'une garantie individuelle qui agit comme correctif ou butoir au libre jeu de l'optimisation.

Pour répondre au mieux au souci de logique et de cohérence de l'ensemble de ses recommandations, la CIPR fait la distinction entre (i) les activités humaines donnant lieu à des expositions: les pratiques qui ajoutent des doses et les interventions qui en retranchent, (ii) les types d'exposition: professionnelle, publique et médicale, et (iii) les expositions réelles et les expositions potentielles. Pour l'application de ses recommandations, elle définit de nombreux niveaux d'action, chacun ayant sa propre signification selon l'activité humaine et le type d'exposition. Elle établit implicitement une échelle de risque pour la gamme de dose dues aux pratiques courantes, en qualifiant les expositions d'inacceptables (au-dessus de la limite), de tolérables (au-dessous de la limite mais au-dessus de la contrainte), d'acceptable (au-dessous de la contrainte) et de négligeable (audessous d'une certaine valeur qui n'est pas précisée).

\footnotetext{
6 Un débat très vif a eu lieu sur le choix de la valeur de $1 \mathrm{mSv}$ qui en fait était déjà introduite dans les recommandations précédentes de la CIPR, comme indiqué plus haut.
} 
Le résultat final constitue un ensemble remarquablement bâti, dont les briques s'intriquent de façon harmonieuse. L'inconvénient majeur est que le système est difficile à appréhender dans son ensemble et que son application est source de confusion et de complications inutiles. Il n'en reste pas moins qu'après sa transcription dans la législation nationale les milieux industriels et médicaux l'adoptent désormais sans état d'âme et respectent ses implications.

\section{Pourquoi changer?}

Dans le milieu des années 90, apparaissent des raisons de modifier le système de protection : (i) des raisons scientifiques, afin de tenir compte de l'apport des dernières connaissances, notamment dans les domaines de la radiobiologie et de l'épidémiologie, (ii) des raisons techniques et pratiques, tirant profit du retour d'expérience et (iii) des raisons sociétales, afin d'adhérer aux courants de pensée adoptés par la société en matière de protection contre les nuisances de tous ordres.

\subsection{Les raisons scientifiques}

Elles concernent :

- Le risque de cancer aux faibles doses, qui est revu à la lumière des données récentes de l'épidémiologie, de la radiobiologie et de l'expérimentation animale. Les principales conclusions sont les suivantes :

- $\quad$ il n'existe aucune raison valable de modifier la valeur de 2 recommandée en 1990 pour le Facteur d'Efficacité de Doses et de Débits de Dose (FEDDD), qui permet de prendre en compte le fait que le risque de cancer aux faibles doses et faibles débits de dose est amoindri par rapport à celui des fortes doses aiguës ;

- l'existence d'un seuil à l'action des rayonnements demeure très improbable, bien qu'il ne puisse pas être formellement écarté dans certaines circonstances ;

- une relation linéaire entre la dose et l'effet constitue la meilleure représentation du risque de cancer radio-induit ;

- certaines valeurs des facteurs de pondération pour les rayonnements $\left(w_{R}\right)$, qui permettent d'apprécier la dangerosité de chaque type de rayonnement, doivent être légèrement modifiées ;

- les valeurs des facteurs de pondération pour les tissus $\left(w_{T}\right)$, qui permettent d'apprécier la dangerosité des rayonnements en fonction de la sensibilité des organes et des tissus, peuvent être affinées ;

- dans la gamme des faibles doses (inférieures à quelques dizaines de mSv), les lésions de l'ADN occupent une position clé dans la cancérogenèse radioinduite. 
Néanmoins, les incertitudes sur les mécanismes et leurs conséquences, particulièrement celles en rapport avec des phénomènes récemment décrits, comme la réponse adaptative, l'instabilité génomique et l'effet de proximité, sont encore trop grandes pour prédire de façon précise comment elles sont susceptibles de modifier l'estimation actuelle du risque des faibles doses extrapolé à partir des études épidémiologiques. Les meilleures estimations du risque, compte tenu de ces restrictions, sont les suivantes :

- risque de cancer mortel (par Sv) : 5,9\% pour la population et 4,6\% pour les travailleurs ;

- risque d'effets héréditaires (par Sv) : 0,2 \% pour la population et $0,1 \%$ pour les travailleurs.

Les valeurs arrondies du détriment total sont de $6,2 \%$ pour la population et de $4,7 \%$ pour les travailleurs, ce qui constitue une légère diminution par rapport aux valeurs précédentes qui étaient respectivement de 7,3\% et 5,6\% (y inclus les cancers non mortels).

- Le risque de maladies non cancéreuses induites par les rayonnements, affectant les systèmes cardiovasculaire, pulmonaire et digestif, qui est confirmé. L'étude des relations dose-effet montre des incertitudes et ne permet pas de se prononcer sur l'existence ou non d'un seuil qui, s'il existe, pourrait se situer aux environs de 500 mSv. La CIPR reconnaît l'importance de ce type d'effets, mais, en l'état actuel des connaissances, est incapable de les inclure dans son estimation du risque global des faibles doses.

- Les effets des fortes doses, qui font l'objet d'un réexamen. La question de la valeur à attribuer à l'EBR (Efficacité Biologique Relative) de chaque rayonnement en fonction de la dose n'est pas entièrement résolue, pas plus que ne l'est la diminution de la nocivité en fonction de la diminution du débit de dose (problème des expositions prolongées pouvant résulter en des doses élevées, situées audessus des seuils des effets déterministes causés par des expositions aiguës).

- Les effets des expositions internes, qui sont difficiles à évaluer, tout au moins sur une base dosimétrique identique à celle utilisée pour les expositions externes. En effet, la distribution spatiale de la dose peut être très hétérogène pour certains rayonnements, comme les émetteurs $\alpha$, les émetteurs $\beta$ mous, les photons de faible énergie et les électrons Auger, particulièrement quand les radionucléides sont déposés dans des régions spécifiques de la cellule ou de l'organe. Dans ce cas, la dose moyennée à l'organe ne peut être représentative du dommage potentiel et sous-estime le risque réel. 
En résumé, les connaissances scientifiques acquises depuis une quinzaine d'années ne justifient ni un remaniement en profondeur du système de protection ni des modifications notables des limites réglementaires; le risque de cancer radio-induit n'est que très faiblement revu à la baisse et la quantification des maladies non cancéreuses et des effets des expositions internes demeure encore incertaine. Mais pour arriver à cette conclusion, il était important de faire le point sur 15 années de résultats de travaux scientifiques. Le débat sur les effets des faibles doses, qui n'a pas perdu de son acuité, conduisait certains à vouloir réviser l'estimation du risque correspondant (à la hausse ou à la baisse).

\subsection{Les raisons techniques et pratiques}

Elles proviennent essentiellement du retour d'expérience. L'application des recommandations de 1990 s'est avérée complexe et un grand nombre de problèmes pratiques a été difficile à résoudre. En fait, les difficultés rencontrées constituent, pour la plupart, l'envers de la médaille de recommandations qui visaient plus la perfection intellectuelle que le pragmatisme. Des exemples de difficultés ou confusions sont fournis par : (i) la frontière entre pratique et intervention, qui n'est pas évidente dans certaines situations durables et/ou anciennes, (ii) la limite de dose pour le public, fixée à $1 \mathrm{mSv}$, difficile à comprendre lorsqu'elle est comparée au bruit de fond naturel, aux différents niveaux d'intervention en cas d'accident ou au niveau de dose acceptable pour le radon domestique (niveaux tous supérieurs à la limite), (iii) les raisons pour lesquelles les limites ne sont pas applicables dans les situations d'urgences, (iv) l'utilisation abusive de la dose collective et certaines de ses conclusions, (v) l'accent mis sur la protection de la collectivité qui peut paraître s'effectuer au détriment de l'individu, etc.

En résumé, ces raisons pourraient sans doute justifier à elles seules une mise à plat des recommandations de 1990, afin de simplifier leur utilisation et d'en supprimer certaines incohérences, qu'elles soient réelles ou apparentes. C'est dans cet esprit de simplification que le Président de la CIPR, Roger Clarke, a présenté au milieu des années 90 de nouveaux concepts sur lesquels il proposait de centrer un nouveau système, comme la dose maîtrisable (" contrôlable») et la dose négligeable ( trivial»), qui permettraient d'échapper à une catégorisation, poussée à l'extrême, des expositions.

\subsection{Les raisons sociétales}

Elles correspondent au besoin d'adapter les règles édictées par la CIPR à l'évolution des courants de pensée de la société moderne. De plus en plus, cette dernière a tendance à centrer ses intérêts sur le seul individu, occultant ainsi l'intérêt général. En outre, la société actuelle accorde une attention grandissante à 
la qualité de son environnement, donc à la protection de la nature. En conséquence, il était normal que la CIPR reconsidère les bases de sa doctrine en choisissant entre : (i) la persistance d'une éthique utilitariste ou un alignement sur une éthique individualiste et (ii) la protection isolée de l'espèce humaine ou la protection de l'ensemble du biotope, en replaçant l'être humain dans le cadre général de son environnement.

- En réponse à la question : protection de la collectivité ou protection de l'individu, la CIPR a estimé qu'elle se devait de renverser la dynamique de son système ; alors que l'approche adoptée en 1990 pouvait se résumer par : si le risque pour la collectivité est maintenu à un niveau acceptable, le risque individuel est aussi à un niveau acceptable, compte tenu de certaines barrières individuelles (limites et contraintes), l'approche actualisée devient : si le risque pour la santé de l'individu le plus exposé est faible, le risque total peut être considéré comme faible, quel que soit le nombre de personnes exposées. Il faut cependant souligner, comme on le verra plus loin, que l'appréciation de la dimension collective du risque n'est pas abandonnée mais sa prise en compte relève davantage du jugement d'expert que du résultat d'une approche mathématique automatique. Il s'agit en fait d'un changement d'ordre éthique, qui fait basculer le système d'un ordre utilitariste à un ordre contractuel (ou pragmatique). En fait, la CIPR avait depuis quelques années recentré son intérêt sur l'individu, en raison notamment de l'émergence de problèmes liés aux personnes et non à la collectivité, comme la sensibilité individuelle d'origine génétique, les indemnisations (maladies professionnelles et séquelles d'accidents) ou plus généralement les conflits entre intérêt général et intérêt particulier.

- En réponse à la question : protection de l'espèce humaine ou protection de l'ensemble du biotope, la CIPR fait le choix de considérer l'ensemble, en situant l'être humain au sein de son environnement. Certes la protection des espèces animales et végétales ne peut pas être calquée directement sur celle de l'espèce humaine, ne serait-ce qu'en raison des importances relatives qu'il convient d'attribuer aux intérêts individuels et collectifs dans les deux domaines. Malgré les lacunes qui existent dans les connaissances, il semble justifié de mettre en place un système consensuel de protection de la nature et de sa bio-diversité, en s'attachant à la sauvegarde du patrimoine naturel. Ce système doit demeurer compatible avec celui de la protection de l'homme en même temps qu'il s'insère dans le contexte général de la toxicologie environnementale. Dans ce domaine comme dans le précédent, les considérations éthiques occupent une place prépondérante dans le choix des options de base. La CIPR souhaite initier une tentative de standardisation de la protection, en mettant en perspective la protection contre les rayonnements, qu'il s'agisse de l'homme ou de la nature, et la protection contre tous les autres agents nocifs, physiques ou chimiques. L'ordre de priorité fixé jusqu'à 
présent - les travailleurs, la population et enfin l'environnement - risque d'être modifié.

- L'importance du dialogue social dans la prise de décisions, particulièrement dans le domaine de la protection de l'être humain et de l'environnement constitue un troisième volet que la CIPR se devait de considérer. La mentalité passéiste qui peut se résumer par « faites-nous confiance puisque nous sommes spécialistes » n'est plus acceptée. La CIPR, jusqu'aux années 80 , édictait ses règles en cercle fermé et décidait «en son âme et conscience» de la frontière entre l'acceptable et l'inacceptable. Lors de l'élaboration de ses recommandations de 1990, elle a révisé timidement son attitude en consultant les grandes organisations internationales et nationales, les sociétés savantes et les partenaires sociaux. Ce processus a conduit à des modifications notables de ses propositions. Le dialogue social généralisé et étendu apparaît aujourd'hui essentiel, qu'il s'agisse de la protection contre les rayonnements ou tout autre agent nocif pour la santé. Il constitue sûrement un pilier de l'acceptabilité d'un certain niveau de risque individuel et collectif. En optant pour la transparence la CIPR a changé ses habitudes, via des consultations répétées auprès des institutions intéressées, qu'elles soient scientifiques, techniques, politiques ou représentatives de divers groupes de pression. En fait, la CIPR n'a rien inventé et n'est même pas avant-gardiste sur ce terrain, puisqu'elle n'a fait que s'aligner sur les autres organisations, en tenant compte des nouveaux modes de gestion des activités à risque.

En résumé, les trois raisons sociétales décrites ci-dessus ont des poids différents dans la justification d'un changement des règles du jeu de la radioprotection : (1) La priorité que la CIPR déclare accorder à l'individu plutôt qu'à la société était déjà sous-jacente dans les recommandations de 1990, qui ont créé implicitement une échelle de risque avec notamment le concept de contrainte, qui s'applique à une source de rayonnements donnée mais concerne l'individu et constitue la borne supérieure pour la sélection des options de protection individuelle ; (2) la décision de considérer la protection de l'espèce humaine dans le cadre de la protection de l'ensemble du biotope constitue une avancée importante, et le fait que la CIPR ait devancé d'autres initiatives prévisibles est un gage de cohérence entre les systèmes de protection des espèces vivantes; (3) l'implication des parties prenantes, particulièrement dans les aspects décisionnels, relève plus du domaine social ou politique, au même titre que le principe de justification, qui constituait le premier pilier du système de 1990 et qui est maintenant jugé largement en dehors du champ des recommandations; il semble donc qu'il y ait ici une contradiction et que la CIPR dans ce cas se préoccupe des règles de fonctionnement des sociétés, dont certaines relèvent des autorités nationales. En conclusion, la protection de l'environnement vivant est le seul point nouveau que la CIPR se devait d'aborder; en revanche, à elle seule, 
elle ne peut justifier un changement radical des règles de protection de l'espèce humaine.

\section{Le projet des nouvelles recommandations}

Depuis 2001, la CIPR travaille sur un projet de nouvelles recommandations, qui se dénomment Recommandations 2005, car destinées en principe à être finalisés en 2005, qui correspond à la fin du mandat des membres actuels ${ }^{7}$. La CIPR note que ces nouvelles recommandations constituent une évolution naturelle et un éclaircissement des précédentes recommandations. Elle rappelle qu'elles doivent assurer un niveau de protection qui doit être considéré comme une obligation et que le non-respect des niveaux de protections indiqués constitue un échec. Les principales modifications qui constituent un démarcage notable par rapport aux recommandations 1990 peuvent se résumer de la façon suivante ${ }^{8}$ :

Les principes de base de protection : Justification des pratiques, Optimisation de la protection et Limitation des expositions, sont conservés mais remaniés dans leur hiérarchie, leur signification et leur application. La CIPR rappelle que la justification, qui par définition ne peut s'appliquer qu'aux situations d'exposition introduites ou poursuivies délibérément, c'est-à-dire aux pratiques donnant lieu à des expositions normales, repose sur des considérations très diverses parmi lesquelles celles relatives à la radioprotection n'occupent qu'une part souvent réduite ; les jugements incombent finalement à des décideurs, souvent au niveau gouvernemental. C'est pourquoi la CIPR, tout en conservant ce principe qui constitue un préalable obligatoire à son système de protection, le juge largement en dehors de sa compétence et de sa responsabilité. La CIPR met l'accent sur le troisième principe, qui devient la Restriction de la dose individuelle en rapport avec une source de rayonnements (la source dominante), qui s'applique désormais avant l'optimisation. Cette modification exprime le souci de la CIPR de focaliser son système de protection sur l'individu et d'en faciliter l'application.

Le niveau fondamental de protection est la restriction appliquée à la dose individuelle résultant d'une source donnée ; c'est la contrainte de dose. Celle-ci doit assurer un niveau de protection aux personnes les plus exposées dans une catégorie d'exposition (professionnelle, publique, médicale), dans toutes les situations relevant du champ d'application des recommandations. Exception faite de l'exposition des patients irradiés pour raisons médicales, la contrainte constitue le niveau de base de protection, qui doit être atteint dans les situations normales,

\footnotetext{
7 La date de publication a été retardée à 2007.

8 D'après le résumé et le texte du projet des Recommandations 2005, présentés sur internet.
} 
TABLEAU I

Contraintes de dose maximales, selon les situations d'exposition, pour une source dominante.

Maximum dose constraints for a dominant source for various exposure situations.

\begin{tabular}{|c|c|c|}
\hline \multirow{2}{*}{$\begin{array}{l}\text { Contraintes } \\
(\mathrm{mSv} \text { en } 1 \text { an })\end{array}$} & \multicolumn{2}{|c|}{ Situations d'exposition } \\
\hline & Caractéristiques des situations & Exemples concrets de situations \\
\hline \multirow{2}{*}{$100 \rightarrow$} & $\begin{array}{l}\text { - Aucun bénéfice } \\
\text { individuel ou } \\
\text { sociétal }\end{array}$ & $\begin{array}{l}\text { - Urgences, uniquement pour sauver des vies } \\
\text { humaines }\end{array}$ \\
\hline & $\begin{array}{l}\text { - Difficilement maittrisable } \\
\text { - Bénéfice individuel direct ou indirect } \\
\text { - Information précise du public, formation } \\
\text { et surveillance des travailleurs }\end{array}$ & $\begin{array}{l}\text { - Urgences } \\
\text { Quand les mesures de protection sont difficiles à } \\
\text { mettre en œuvre }\end{array}$ \\
\hline $20 \rightarrow$ & $\begin{array}{l}\text { - Facilement maitrisable } \\
\text { - Bénéfice individuel direct ou indirect } \\
\text { - Information et évaluation de l'exposition } \\
\text { du public, formation et surveillance des } \\
\text { travailleurs }\end{array}$ & $\begin{array}{l}\text { - Exposition professionnelle en général } \\
\text { - Expositions préexistantes } \\
\text { - Exposition des personnes du public dans les } \\
\text { urgences, quand les mesures de protection sont } \\
\text { faciles à mettre en œuvre } \\
\text { - Exposition des accompagnateurs et soignants } \\
\text { de patients (domaine médical) }\end{array}$ \\
\hline $1 \rightarrow$ & $\begin{array}{l}\text { - Facilement maîtrisable } \\
\text { - Bénéfice sociétal, mais } \\
\text { pas de bénéfice individuel direct } \\
\text { - Information générale, pas de formation, } \\
\text { pas d'évaluation } \\
\text { individuelle de l'exposition }\end{array}$ & - Exposition des personnes du public en général \\
\hline $0,01 \rightarrow$ & $\begin{array}{l}\text { - Utilité du contrôle sujette } \\
\text { à appréciation }\end{array}$ & - Toutes les situations d'expositions \\
\hline
\end{tabular}

les accidents et les urgences, ainsi que dans le cas d'exposition maîtrisable préexistante. La contrainte de dose ne représente pas une démarcation entre la zone des « doses dangereuses » et celle des « doses sans danger». Un dépassement de la contrainte de dose est toujours synonyme d'échec et peut même constituer une infraction répréhensible, si les autorités nationales lui ont conféré ce statut.

La CIPR indique quatre valeurs maximales de contraintes (exprimées en dose efficace), pour les travailleurs et les personnes du public, regroupant plusieurs situations d'exposition à une source, prédominante sur toutes les autres sources. Le tableau I résume les caractéristiques des situations répondant à ces critères et fournit des exemples concrets. Ces valeurs maximales ont pour but d'aider les autorités nationales dans le choix des contraintes réglementaires ou non ; ces dernières sont évidemment inférieures aux valeurs maximales indiquées plus haut ; la CIPR estime que, raisonnablement, la réduction ne devrait pas dépasser un facteur 10 . 
Les contraintes de dose doivent être complétées par une optimisation de la protection. Cette démarche est rendue nécessaire du fait de l'existence d'une certaine probabilité d'effets néfastes sur la santé, quel que soit le niveau de l'exposition reçue en plus de l'exposition d'origine naturelle. C'est la raison pour laquelle la CIPR recommande que des mesures supplémentaires, plus strictes que celles qui conduisent à la détermination des contraintes de dose, soient envisagées pour chaque source de rayonnements. Cette exigence d'optimisation, implique, comme par le passé, que toutes les expositions soient aussi basses que raisonnablement possible, compte tenu des facteurs économiques et sociaux. Cette exigence ne peut pas s'exprimer en termes quantitatifs de portée générale; les opérateurs et les autorités nationales responsables de la protection doivent porter des jugements spécifiques à chaque situation cause de l'exposition d'individus. En fait, l'optimisation de la protection va plus loin que la simple réduction des doses reçues par les travailleurs et le public dans des situations normales, car elle doit s'intéresser aussi à la prévention des accidents et à toutes les autres sources d'expositions potentielles.

Le niveau de protection défini pour un individu vis-à-vis de toutes les sources, dans une catégorie d'exposition donnée et dans les seules situations normales, est la limite de dose. Il est rare qu'il soit possible d'évaluer l'exposition totale d'une personne due à toutes les sources maîtrisables. La comparaison de cette exposition totale et de la limite, effectuée afin de vérifier le respect de la réglementation, ne peut donc être qu'approximative; cette approximation est moindre pour l'exposition des travailleurs que pour celle des personnes du public. La CIPR estime que les limites de dose (dose efficace) recommandées en 1991 dans sa Publication 60 (ICRP, 1991) procurent un niveau suffisant de protection dans les situations normales. En conséquence, elle maintient pour les travailleurs la limite de $20 \mathrm{mSv}$ par an (moyennée sur 5 ans, soit $100 \mathrm{mSv}$ en 5 ans) et pour le public celle de $1 \mathrm{mSv}$ (pouvant atteindre certaines années $5 \mathrm{mSv}$ dans la mesure où la moyenne sur 5 ans ne dépasse pas $1 \mathrm{mSv}$ par an). Il convient de rappeler qu'à l'inverse de la contrainte la limite de dose n'a aucun sens en dehors des situations normales, par exemple dans les situations accidentelles.

En résumé, les principes généraux qui assurent aux personnes le niveau requis de protection reposent en premier lieu sur les contraintes (relatives à une source donnée de rayonnements) assujetties à l'optimisation, qui s'appliquent quelle que soit la situation, et secondairement sur les limites (relatives à l'ensemble des sources d'exposition individuelle) qui ne s'appliquent qu'aux situations normales.

La culture de sûreté apparaît pour la première fois dans des recommandations générales de la CIPR ; elle est considérée comme un corollaire du processus d'optimisation, qui doit l'encourager et la susciter. Les responsables de la 
radioprotection doivent régulièrement se poser la question : ai-je fait tout ce qui est raisonnablement en mon pouvoir pour éviter ou réduire les doses? Cette interrogation, qui implique une part d'appréciation, nécessite, pour être résolue de façon satisfaisante, la coopération de toutes les parties impliquées, au minimum constituées par les responsables opérationnels et les autorités.

L'engagement des parties prenantes constitue aussi une nouveauté pour des recommandations de la CIPR. Selon cette dernière, la démarche d'optimisation doit s'effectuer avec le concours de toutes les parties impliquées dans une conjoncture donnée qui les inquiète en raison du danger d'éventuelles conséquences. L'expérience prouve que cet engagement permet (i) l'intégration de nombreux paramètres dans les prises de décision, (ii) l'amélioration de la qualité finale des décisions, (iii) la résolution de conflits d'intérêts contradictoires, (iv) l'établissement d'un état de confiance envers les institutions, ainsi que (v) l'éducation des professionnels et du public. En outre, l'engagement des parties prenantes renforce la culture de sûreté et permet d'introduire une certaine souplesse dans la gestion du risque radiologique, garante de décisions efficaces et durables.

L'exclusion de sources de rayonnements en dehors du champ d'application des recommandations de la CIPR s'applique aux sources qui délivrent des doses efficaces annuelles très basses et qui sont souvent difficiles ou impossibles à maîtriser. L'actuel projet recommande les niveaux d'exclusion suivants, exprimés en concentration d'activité : pour les émetteurs $\alpha$ artificiels : $0,01 \mathrm{~Bq}$ par gramme ; pour les émetteurs $\beta$ et $\gamma$ artificiels: $0,1 \mathrm{~Bq}$ par gramme ; pour les radionucléides naturels situés au début des chaines de désintégration, par exemple l'uranium-238 et le thorium-232:1,0 Bq par gramme, ou encore le-potassium $40: 10 \mathrm{~Bq}$ par gramme. Ces valeurs d'exclusion constitueraient les bornes au-dessus desquelles les radionucléides seraient considérés comme des produits radioactifs.

L'exclusion constitue sans doute le chapitre des nouvelles recommandations le plus contesté. Un des points principaux de la contestation met en cause le jugement par lequel le risque associé à une dose est considéré comme négligeable. En fait, la décision fait appel à un jugement de valeur, dès lors que la relation dose-effet est supposée linéaire et sans seuil. La discussion est semblable à celles du passé au sujet du concept "de minimis », qui reposait sur l'argument suivant : le risque peut être ignoré puisqu'il est petit. En fait, un risque négligeable n'est pas nécessairement acceptable (acceptable pour qui ?) et un risque acceptable n'est pas nécessairement négligeable ${ }^{9}$. On peut donc s'attendre à ce que les

9 D'après Bo Lindell, Président de la CIPR de 1977 à 1985, dans : How safe is safe enough ? (Lauriston Taylor lectures in Radiation Protection and Measurements Lecture $N^{\circ} 12$, Bethesda, MD, NCRP, 1988. 
propositions qui figurent dans l'actuel projet soient amendées dans le prochain projet.

Les grandeurs dosimétriques sont simplifiées, en raison des nombreuses confusions qui ont eu lieu entre les trois grandeurs utilisées précédemment : dose équivalente, équivalent de dose et dose efficace, toutes exprimées par la même unité le sievert. La CIPR propose de supprimer le terme «dose équivalente » (combinaison de la dose absorbée et du facteur de pondération pour les rayonnements $w_{R}$ ) et de le remplacer par celui de dose pondérée pour les rayonnements, en attendant de lui trouver une meilleure appellation. La définition de la dose efficace demeure inchangée : c'est la dose absorbée multipliée par les deux facteurs de pondération $\mathrm{w}_{\mathrm{R}}$ pour les rayonnements et $w_{T}$ pour les tissus. La CIPR fournit de nouvelles valeurs pour ces deux facteurs de pondération, en raison d'avancées dans les connaissances. Le facteur de pondération pour les rayonnements $w_{R}$ prend les valeurs suivantes : 1 pour les photons de toutes énergies, 1 pour les électrons et muons de toutes énergies, 2 pour les protons autres que les protons de recul (d'énergie supérieure ou égale à $2 \mathrm{MeV}$ ), 20 pour les émetteurs $\alpha$, les fragments de fission et les noyaux lourds ; une courbe lissée est recommandée pour les neutrons en fonction de leur énergie. Le facteur de pondération pour les tissus $w_{T}$ est plus profondément remanié que le précédent, sur la base de nouvelles données épidémiologiques, qui ont permis d'allonger la liste tout en la simplifiant. Les nouvelles valeurs de $w_{T}$ sont regroupées dans quatre catégories : 0,12 pour la moelle osseuse, le colon, les poumons et l'estomac et pour un regroupement de tissus divers ; 0,08 pour le sein et les gonades, 0,05 pour la vessie, l'œsophage, le foie et la thyroïde ; 0,01 pour la surface osseuse, le cerveau, les glandes salivaires et la peau.

La protection des espèces non humaines est le dernier volet réellement novateur des recommandations 2005. Il était annoncé par la Publication 91 sur la protection de l'environnement (ICRP, 2004), dont les grandes lignes sont reprises dans le projet des futures recommandations. La CIPR indique que le nouveau système est conçu pour constituer un ensemble harmonieux et cohérent de la protection de l'ensemble des espèces vivantes, humaine, animales et végétales. Elle ajoute qu'elle a l'intention de constituer des jeux de répertoires et modèles dosimétriques de référence, ainsi que des données permettant d'apprécier les relations exposition-dose et dose-effet; l'interprétation des résultats sera développée pour un nombre limité d'animaux et de plantes types. Cet ensemble permettra de garantir que la protection des humains et celle des autres organismes vivants reposent sur les mêmes bases scientifiques, particulièrement en ce qui concerne les relations exposition-dose et dose-effet au niveau de la molécule, de la cellule, du tissu, de l'organe et de l'organisme dans son ensemble. La CIPR espère être en mesure de proposer pour les animaux et les plantes, par analogie au système 
pour l'être humain qui repose sur la contrainte de dose, un ensemble de « niveaux de considération dérivés » pour l'environnement ambiant, qui pourrait constituer une base pour des normes internationales, dont le besoin devient de plus en plus fort.

\section{Les conséquences pratiques du changement}

Les conséquences de ces modifications sont essentiellement d'ordre conceptuel. Les nouvelles recommandations se distinguent de celles de 1990 par deux caractéristiques principales : simplification du système et adaptation au courant de pensée moderne.

La simplification réside dans la création d'un système nettement plus général et moins compartimenté que le précédent, qui distinguait les pratiques et les interventions, les expositions réelles existantes et les expositions potentielles, les catégories d'expositions, etc. Ces distinctions étaient en grande partie dues au choix du concept de limite comme base du système de restriction des doses. Les recommandations 2005 choisissent le concept de contrainte de dose comme niveau fondamental, indépendamment du type d'exposition et de la situation. Le contrôle s'effectue dorénavant sur la dose due à la source d'exposition principale (dominante) et non sur la dose totale due à l'ensemble des sources. En outre et surtout, la contrainte 2005 a une portée beaucoup plus large que la limite 1990. Elle permet de gérer les différentes situations d'exposition à l'aide d'une échelle unique comportant seulement quatre valeurs de contraintes de dose. Ces quatre valeurs ne remplacent pas l'ensemble des niveaux de référence, d'action, d'investigation, d'intervention, etc. (actuellement au nombre d'environ 25), à l'origine de nombreuses confusions. La nouvelle présentation se borne à placer au second plan les anciens niveaux opérationnels mais elle les situe dans une rationalité globale associée aux situations d'exposition. Elle présente l'avantage de mettre en évidence le continuum des risques et leur appréciation en fonction des caractéristiques de l'exposition (maîtrise, bénéfice individuel, information...). En plus de son intérêt simplificateur, la contrainte, en tant qu'outil fondamental du système de protection, présente l'avantage de rendre aux autorités leur légitimité dans le choix final des valeurs opérationnelles des doses pouvant ou non être reçues par les professionnels ou les personnes du public.

Le nouveau système ne mentionne qu'accessoirement l'ancien premier principe des recommandations 1990, la Justification des pratiques. Ce principe repose sur des considérations d'ordres très variés, social, économique et politique ; le poids des considérations sanitaires est souvent négligeable en comparaison avec les précédentes, parfois inexistant. Il est cependant important de continuer d'y faire référence. 
La simplification s'applique aussi aux grandeurs dosimétriques. Aux trois grandeurs de 1990, dose absorbée, dose équivalente et dose efficace, succèdent la même dose absorbée, qui constitue l'indicateur de gravité primaire pour les effets tissulaires (correspondants aux effets déterministes), et la dose efficace, qui constitue l'indicateur de gravité pour les effets stochastiques. La dose équivalente de 1990 ne sert qu'à exprimer le risque pour un organe ou un tissu particulier ; elle change de nom (provisoirement dose pondérée pour les rayonnements), afin de montrer l'usage limité qui doit en être fait.

L'adaptation au courant de pensée moderne donne la prédominance à la protection de l'individu, en supposant que, si cette dernière est performante, la collectivité est protégée à des niveaux de protection équivalents. Les efforts de protection se focalisent sur la source de rayonnements affectant l'individu de façon prédominante (source-related), et non plus sur l'individu affecté par l'ensemble des sources de son environnement (individual-related). Il en résulte que les restrictions s'appliquent dorénavant à la source et non à l'individu. Cette approche, située à l'opposé de la doctrine passée de la CIPR, devrait conduire elle aussi à une grande simplification des pratiques quotidiennes de la radioprotection.

Cette adaptation se traduit aussi par le parti pris d'ajouter de nouveaux volets au système : inclusion de la culture de sûreté dans le processus d'optimisation de la protection, implication des parties prenantes dans les prises de décisions importantes pour la protection des humains et de leur environnement, et protection des espèces non humaines harmonisée avec celle proposée pour les être humains.

\section{Conclusions}

Les nouvelles recommandations, en plus d'un effort évident de clarifications de la doctrine de protection contre les rayonnements ionisants, présentent trois caractéristiques principales qui devraient constituer des avantages notables par rapport à celles de 1990 : (i) elles sont faciles à comprendre et simples à appliquer, (ii) au plan de la pratique quotidienne et des répercussions sur la conception et l'utilisation des installations, elles ne devraient pas nécessiter des adaptations notables ou des changements importants de comportement de la part des praticiens de la protection, des exploitants et des autorités responsables (par exemple, la limite de dose est inchangée), et (iii) elles démontrent la volonté de s'adapter au mode de pensée et à la culture modernes.

La priorité accordée maintenant à l'individu par rapport à la collectivité n'apportera rien de particulièrement nouveau du point de vue opérationnel. Il officialise le changement progressif de comportement sociétal vis-à-vis des 
risques de la vie. Son intérêt ne réside sans doute pas dans le choix délibéré de la priorité de l'individu sur la collectivité, mais sur certaines restrictions pratiques, qui devraient s'ensuivre ipso facto, comme celle de l'usage abusif de la dose collective, source d'interprétations souvent erronées.

Le projet tel qu'il est présenté actuellement constitue en fait une actualisation des recommandations 1990. En raison de sa concision, il ne peut être compris et assimilé que par des lecteurs qui possèdent une culture solide et approfondie de la radioprotection moderne. Les recommandations 1990 comportaient des volets explicatifs et pédagogiques détaillés, qui font défaut dans ce projet. Ces aspects seront développés dans des « documents fondateurs » en cours d'élaboration et qui seront publiés en même temps que les nouvelles recommandations générales ${ }^{10}$. C'est pour permettre la publication concomitante de tous ces documents que celleci est retardée à 2006 (voire début 2007). Au stade actuel, certaines rubriques des nouvelles recommandations, particulièrement celles qui traitent de nouveautés, devraient être développées et étayées par une argumentation solide ; d'autres sont difficiles à comprendre et apparaissent parfois contradictoires. Il paraîtrait normal, par exemple, d'expliquer pourquoi la CIPR accorde une place plus réduite au principe de Justification des pratiques, de montrer clairement la différence entre les contraintes 2005 et 1990, de préciser les significations et utilisations respectives de la contrainte et de la limite, de développer le concept d'exclusion et d'en justifier le niveau, etc.

En résumé, la finalisation du projet de recommandations exige encore beaucoup de travail, plus au plan de la présentation et de l'explication qu'à celui de la conception du système proposé. C'est la raison pour laquelle le document est proposé pour avis à une très large audience, spécialisée dans des secteurs extrêmement variés ; il faut espérer que les observations seront constructives et considérées favorablement par la CIPR.

Un dernier point peut paraître critiquable : c'est la partie modeste qui est dévolue au domaine médical (10 paragraphes sur 251). Dans les années 60 et suivantes, qui ont vu le développement de l'industrie nucléaire, la CIPR s'est logiquement focalisée de façon prédominante sur le domaine industriel et les situations accidentelles. Aujourd'hui il peut paraitre curieux que ses nouvelles recommandations n'accordent qu'une place extrêmement réduite à la protection en médecine. Cette réserve ne doit pas être interprétée comme un message selon lequel les problèmes qui relèvent du domaine médical seraient actuellement résolus ; cette assertion serait en contradiction avec l'attitude présente de la CIPR,

${ }^{10}$ Ces documents ont été accéssibles pour commentaires sur le site de la CIPR au cours de la période mai-juillet 2005. 
qui montre depuis les années 90 son intérêt croissant pour les problèmes médicaux, comme le prouvent les très nombreuses publications récentes qui lui sont consacrées (depuis 1996 avec la Publication 73 sur la protection en médecine, sept publications traitent de questions pratiques et quatre autres sont à paraître prochainement). Les raisons en sont plutôt à rechercher dans le fait que le concept de contrainte, mis en avant dans les nouvelles recommandations, n'est pas très approprié pour la protection du patient. Il convient cependant de noter que, dans le cadre de la rencontre entre la Commission principale de la CIPR et les professionnels de la radioprotection organisée par la SFRP en marge de la réunion de la Commission principale en mars 2005 à Paris, le président de la CIPR, en réponse aux remarques du groupe de travail SFRP/CIPR (Schieber et Cordoliani, 2005), a indiqué que cette question sera examinée avec attention avant la publication des nouvelles recommandations.

\section{RÉFÉRENCES}

ICRP publication 1 (1959) Recommendations of the International Commission on Radiological Protection. Pergamon Press, Oxford. Superseded by ICRP Publication 26.

ICRP publication 2 (1960) Report of Committee II on Permissible Dose for Internal Radiation. Pergamon Press, Oxford. Superseded by ICRP Publication 30.

ICRP publication 9 (1963) Recommendations of the International Commission on Radiological Protection. Pergamon Press, Oxford. Revision of ICRP Publication 6, superseded by ICRP Publication 26

ICRP publication 22 (1973) Implication of Commission Recommendations that Doses be Kept as Low as Readily Achievable. Pergamon Press, Oxford.

ICRP publication 26 (1977) Recommendations of the International Commission on Radiological Protection, Ann. ICRP 1(3). Superseded by ICRP Publication 60.

ICRP publication 60 (1991) 1990 Recommendations of the International Commission on Radiological Protection, Ann. ICRP 21(1-3).

ICRP publication 73 (1996) Radiological Protection and Safety in Medicine, Ann. ICRP 26(1).

ICRP publication 91 (2004) A framework for assessing the impact of ionising radiation on non-human species. Elsevier. Traduite en français sous le titre : Cadre méthodologique pour évaluer l'impact des rayonnements ionisants sur les espèces non humaines, à paraître en 2005.

Schieber C., Cordoliani Y.-S. (2005) Principales remarques du GT SFRP/CIPR concernant le projet de recommandations 2005 de la CIPR, Radioprotection 40, 89-94. 